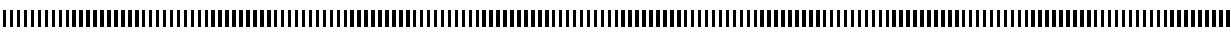

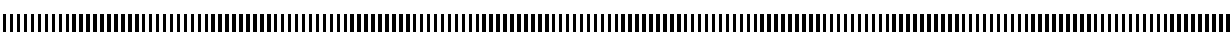
| | | |

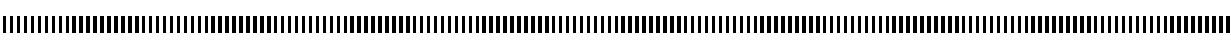

\title{
Théorie générale d'équation de type hyperbolique-parabolique non linéaire
}

\author{
Hamidou Toure \\ Laboratoire d'Analyse Mathématique des Equations (LAME) \\ UFR Sciences Exactes et Appliquées (UFR-SEA) \\ Université de Ouagadougou Bp: 7021 Ouagadougou, Burkina Faso. \\ toureh@univ-ouaga.bf
}

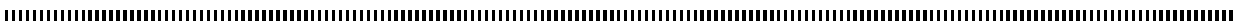

RÉSUMÉ. Nous développons une théorie générale pour des équations d'évolution de type hyperboliqueparabolique non linéaire à l'aide de la théorie des semi-groupes non linéaires dans les espaces de Banach. Nous établissons des résultats d'existence, d'unicité et de dependance continue par rapport aux données d'une bonne solution du problème de Cauchy ou des problèmes aux limites associées à cette équation sous des hypothèses très générales.

Avec des hypothèses complementaires, nous montrons que cette bonne solution est une solution locale de type entropique, nous étudions également l'unicité des solutions faibles et l'existence de solution forte.

ABSTRACT. We develop general theory for degenerate hyperbolic-parabolic type problems using semi-group theory in Banach spaces. We establish existence, uniqness results and continuous dependance with respects to data for mild solution.

Similar results are developped for weak solution of entropy type, and existence of solutions are studied.

MOTS-CLÉS : Equation parabolique degénérée, solution faible, semi-groupe, solution entropique

KEYWORDS : Degenerate parabolic equation, weak equation, entropy solution, semi-group

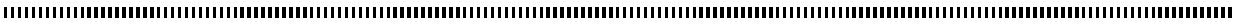




\section{Introduction}

Nous présentons dans ce travail divers aspects mathématiques de problèmes paraboliques fortement degénérés en une dimension d'espace.

Il s'agit d'équations d'évolution modélisant par exemple l'écoulement d'un fluide dans un milieu poreux ou bien décrivant les effets combinés de diffusion non linéaire et de convection de matière.

Le problème considérée est de la forme :

$$
\begin{cases}\frac{\partial u}{\partial t}=\varphi(u)_{x x}-\psi(u)_{x}+f & \operatorname{sur} Q=] 0, T[\times I, \\ u=0 & \left.\operatorname{dans} \sum=\right] 0, T[\times \partial I, \\ u(0)=u_{0} & \operatorname{sur} I\end{cases}
$$

où $I$ est un ouvert de $\mathbb{R}, f$ est donnée sur $Q=] 0, T\left[\times I\right.$ et $u_{0}$ sur $I$. $\varphi$ et $\psi$ sont des fonctions continues avec $\varphi$ croissante au sens large. Par la théorie des semi-groupes non linéaires dans $L^{1}(I)$, on introduit une notion de $<<$ bonne solution $>>$ du problème. On introduit tout d'abord une notion de solution entropique du problème elliptique associé :

$$
(P S)\left\{\begin{array}{lc}
-\varphi(u)_{x x}+\psi(u)_{x}=f & \text { sur } I \\
u=0 & \text { sur } \partial I .
\end{array}\right.
$$

Nous avons introduit dans [2] une notion de solution entropique du problème elliptique associé $(P S)$.

Définition 1 On appelle solution entropique du problème $(P S)$ toute fonction

$u \in L_{\text {loc }}^{1}(\mathbb{R}) \cap L_{\text {loc }}^{\infty}(\mathbb{R}), u=0$ p.p sur $\mathbb{R} \backslash I, \varphi(u) \in \mathcal{C}(\mathbb{R})$. Il existe $h \in C(\mathbb{R})$ tel que $h=-\varphi(u)_{x}+\psi(u)$ dans $\mathcal{D}^{\prime}(I)$ et

$$
\int_{\mathbb{R}} \operatorname{sign}(u-k)\left\{[h-\psi(k)] \zeta_{x}+f \zeta\right\} d x \geq 0 \quad \forall \zeta \in D^{+}(\mathbb{R}), \quad k \in \mathbb{R} .
$$

Notons

$$
L_{0}^{1}(I)=\left\{u \in L^{1}(\mathbb{R}), u=0 \text { p.p sur } \mathbb{R} \backslash I\right\} .
$$

Etant donné $x \in \mathbb{R}$, on note $u(x+)$ la limite à droite et $u(x-)$ la limite à gauche lorsqu'elles existent.

Proposition 1 Soient $f \in L_{l o c}^{1}(\mathbb{R})$ et $u \in L_{\text {loc }}^{1}(\mathbb{R})$ verifiant pour tout $x \in \mathbb{R}, u(x+)$ et $u(x-)$ existent. Alors u est solution entropique de $(P S)$ si et seulement si u est solution au sens suivant :

$h=-\varphi(u)_{x}+\psi(u)$ dans $\mathcal{D}^{\prime}(I)$ et pour tout $x \in \mathbb{R}$ et $k \in[\underline{u}(x), \bar{u}(x)]$, on a

$u=0 p \cdot p$

$[u(x+)-$

où on a noté $\underline{u}(x)=u(x+) \wedge u(x-)$ et $\bar{u}(x)=u(x+) \vee u(x-)$.

Nous associons au problème $(P S)$ un opérateur défini sur $L_{0}^{1}(I)$ par $A=A_{\varphi, \psi}$, $A u=f$ si $u \in L_{0}^{1}(I), f \in L_{0}^{1}(I)$ et $u$ est solution entropique de $(P S)$. 
Théorème 1 L'opérateur A est unique et vérifie les propriétés suivantes :

1) A est fortement $T$ - accrétif dans $L_{0}^{1}(I)$

2) $\forall \lambda>0, J_{\lambda}=(I+\lambda A)^{-1}, D_{\lambda}=R(I+\lambda A)$

$$
D\left(J_{\lambda}\right) \supset B V(\mathbb{R}) \cap L_{0}^{1}(I) \text { et } J_{\lambda}\left(B V(\mathbb{R}) \cap L_{0}^{1}(I)\right) \subset B V(\mathbb{R}),
$$

3) $D(A) \cap B V(\mathbb{R})$ est dense dans $L_{0}^{1}(I)$.

A l'aide de la théorie générale des équations d'évolution dans les espaces de Banach, nous en avons déduit l'existence et l'unicité de solution semi-groupe du problème :

$$
\left\{\begin{array}{l}
\frac{d u}{d t}+A u=f, \\
u(0)=u_{0},
\end{array}\right.
$$

pour toutes données $f \in L^{1}(Q)$ et $u_{0} \in L_{0}^{1}(I)$.

Nous avons établi la dépendance continue de ses solutions par rapport aux données $f, u_{0}, \varphi$ et $\psi$.

Notons qu'une étude de l'opérateur dans $L^{1}$ avait été effectuée par Wu Zhuogun avec une définition différente exigeant des hypothèses restrictives sur $\varphi$ et $\psi$.

\section{Théorie locale}

Considérons l'équation quasi-linéaire $E(\psi, \varphi, f, Q)$

$$
\frac{\partial u}{\partial t}=\varphi(u)_{x x}-\psi(u)_{x}+f \operatorname{sur} Q
$$

où $Q$ un ouvert de $\mathbb{R}^{2}, \psi, \varphi$ des fonctions continues de $\mathbb{R}$ dans $\mathbb{R}$ avec $\varphi$ croissante au sens large.

On fait la normalisation $\varphi(0)=\psi(0)=0$.

Precisons tout d'abord la notion de solution classique de $(E)$. On appelle solution classique de $E(\psi, \varphi, f, Q)$ toute fonction $u \in \mathcal{C}(Q)$ telle que :

$$
\begin{aligned}
u_{t} & =\varphi(u)_{x x}-\psi(u)+f \text { dans } Q, \\
\text { où } u_{t} & =\frac{\partial u}{\partial t}, \varphi(u)_{x}, \varphi(u)_{x x} \in \mathcal{C}(Q) .
\end{aligned}
$$

Nous avons alors que $f \in \mathcal{C}(Q)$.

Notons que lorsque $\psi, \varphi \in \mathcal{C}(Q), f \in \mathcal{C}(Q)$ : toute fonction $u \in \mathcal{C}^{1}(Q)$ telle que

$$
u_{t}=\varphi(u)_{x x}-\psi(u)+f \text { dans } \mathcal{D}^{\prime}(Q)
$$

est solution classique de $(E)$.

Définition 2 On appelle solution généralisée de $E(\psi, \varphi, f, Q)$ toute fonction $u \in L_{l o c}^{1}(Q)$ verifiant: Il existe une suite

$$
\begin{aligned}
& \left(\psi_{n}, \varphi_{n}, f_{n}, u_{n}\right) \text { de } \mathcal{C}(\mathbb{R}) \times \mathcal{C}_{m}(\mathbb{R}) \times \mathcal{C}(Q)^{2} \text { avec } u_{n} \text { solution classique de } \\
& E_{n}=E\left(\psi_{n}, \varphi_{n}, f_{n}, Q\right) \text { telle que }
\end{aligned}
$$




$$
\left\{\begin{array}{l}
u_{n} \longrightarrow u ; f_{n} \longrightarrow f \text { dans } L_{l o c}^{1}(Q), \\
\psi_{n} \longrightarrow \psi ; \varphi_{n} \longrightarrow \varphi \text { dans } \mathcal{C}(\mathbb{R}), \\
\psi_{n}\left(u_{n}\right) \longrightarrow \psi(u) ; \varphi_{n}\left(u_{n}\right) \longrightarrow \varphi(u) \text { dans } L_{\text {loc }}^{1}(Q)
\end{array}\right.
$$

où on a noté $\mathcal{C}_{m}(\mathbb{R})$ l'ensemble des fonctions de $\mathbb{R}$ dans $\mathbb{R}$ continues et monotones croissante (au sens large).

Remarque 1 Soit $\left(\psi_{n}, \varphi_{n}, f_{n}, u_{n}\right)$ une suite de $\mathcal{C}(\mathbb{R}) \times \mathcal{C}_{m}(\mathbb{R}) \times L_{\text {loc }}^{1}(Q)^{2}$ convergente vers $(\psi, \varphi, f, u)$ au sens de (9). Si pour tout $n, u_{n}$ est solution gééralisée de $E\left(\psi_{n}, \varphi_{n}, f_{n}, Q\right)$ alors u est solution généralisée de $E(\psi, \varphi, f, Q)$.

La notion de solution généralisée est une notion globale, il lui correspond la notion locale suivante

Définition 3 On appelle solution généralisée locale de $E(\psi, \varphi, f, Q)$ toute fonction $u \in$ $L_{\text {loc }}^{1}(Q)$ vérifiant : tout point $\left(t_{0}, x_{0}\right) \in Q$, admet un voisinage ouvert $Q_{1}$ de $Q$ telle que la restriction de u à $Q_{1}$ soit une solution généralisée de $E\left(\psi, \varphi, f, Q_{1}\right)$.

Suivant S. N. Kruskhov, on définit la solution entropique de $E(\psi, \varphi, f, Q)$

On appelle solution entropique de $E(\psi, \varphi, f, Q)$, toute fonction $u \in L_{\text {loc }}^{1}(Q)$ vérifiant : $\psi(u), \varphi(u) \in L_{\text {loc }}^{1}(Q)$ et pour tout $k \in \mathbb{R}$, on a :

$$
\begin{aligned}
\frac{\partial}{\partial t}|u-k|+\frac{\partial}{\partial x}\left(\operatorname{sign}_{0}(u-k)[\right. & \psi(u)-\psi(k)]) \leq \operatorname{sign}_{0}(u-k) f \\
+ & \frac{\partial^{2}}{\partial x^{2}}|\varphi(u)-\varphi(k)| \operatorname{dans}^{\prime}(Q),
\end{aligned}
$$

où $\operatorname{sign}_{0} r=\frac{r}{|r|}$ pour $r \neq 0$, et 0 sinon.

Proposition 2 Toute solution généralisée locale de $E(\psi, \varphi, f, Q)$ est solution entropique.

On suppose dans ce qui suit que $Q=] 0, T[\times \mathbb{R}$ avec $t>0$.

On se donne $u_{0} \in L^{\infty}(\mathbb{R})$ et $f \in L_{l o c}^{1}(Q)$ verifiant pour $\left.p . p t \in\right] 0, T[$,

$f(t) \in L^{\infty}(\mathbb{R}), \int_{0}^{T}\|f(t)\|_{L^{\infty}(\mathbb{R})} d t<\infty$.

On considère le problème de Cauchy, $P C\left(\psi, \varphi, f, u_{0}\right)$

$$
(P C) \begin{cases}\frac{\partial u}{\partial t}=\varphi(u)_{x x}-\psi(u)_{x}+f & \operatorname{sur} Q, \\ u(0)=u_{0} & \text { sur } \mathbb{R} .\end{cases}
$$

On appelle solution généralisée (resp. généralisée locale) du problème de Cauchy $(P C)$, toute solution généralisée (resp. généralisée locale) $u(t) \longrightarrow u_{0}$ dans $L_{l o c}^{1}(\mathbb{R})$ lorsque $t \longrightarrow 0$ essentiellement.

Théorème 2 Il existe une unique fonction $u \in L^{\infty}(Q)$ solution généralisée locale de $(P C)$; de plus $u \in \mathcal{C}\left([0, T] ; L_{\text {loc }}^{1}(\mathbb{R})\right)$ et vérifie

$$
\|u(t)\|_{L^{\infty}(\mathbb{R})} \leq\left\|u_{0}\right\|_{L^{\infty}(\mathbb{R})}+\int_{0}^{T}\|f(t)\|_{L^{\infty}(\mathbb{R})} d t
$$

pour tout $t \in[0, T]$ et u est solution généralisée de $(P C)$. 


\section{Problème elliptique- parabolique non linéaire}

On se donne $b$ et $\varphi$ des fonctions de $\mathbb{R}$ dans $\mathbb{R}$ continues croissantes ( au sens large ). On suppose de plus que $b$ est surjective.

Soit d'autre part $a(k, \zeta)$ une fonction de $\mathbb{R} \times \mathbb{R}$ dans $\mathbb{R}$ continue croissante au sens large en $\zeta$.

On fait l'hypothèse de coercivité suivante sur $a$ :

$$
\lim _{|\zeta| \longrightarrow+\infty} \inf _{|k| \leq R}|a(k, \zeta)|=+\infty, \forall R>0 .
$$

Nous avons introduit la notion de solution entropique du problème elliptique (au sens de Bénilan)

$$
\left.(P S) \quad b(u)-a\left(u, \varphi(u)_{x}\right)_{x}=f \quad \text { sur }\right] 0, T[\times \mathbb{R}
$$

associé au problème d'évolution (PE)

$$
(P E) \begin{cases}b(u)_{t}-a\left(u, \varphi(u)_{x}\right)_{x}=f & \text { sur }] 0, T[\times \mathbb{R} \\ b(u(0))=v_{0} & \text { sur } \mathbb{R}\end{cases}
$$

pour $f$ donnée sur $Q$ et $v_{0}$ sur $\mathbb{R}$.

Définition 4 Soit $f \in L_{\text {loc }}^{1}(\mathbb{R})$, on appelle solution entropique de (PS), toute fonction $u \in L^{\infty}(\mathbb{R})$ telle que $\varphi(u) \in W^{1, \infty}(\mathbb{R})$ et il existe $h \in \mathcal{C}(\mathbb{R})$, $h=a\left(u, \varphi(u)_{x}\right)$ p.p $x \in \mathbb{R}$ verifiant

$$
\int_{\mathbb{R}} \operatorname{sign}_{0}^{+}(b(u)-b(k))\left\{(H(k)-h) \xi_{x}+(f-b(u)) \xi\right\} d x \geq 0
$$

et

$$
\int_{\mathbb{R}} \operatorname{sign}_{0}^{+}(b(k)-b(u))\left\{(H(k)-h) \xi_{x}+(f-b(u)) \xi\right\} d x \leq 0
$$

pour tout $k \in \mathbb{R}, \xi \in \mathcal{D}(\mathbb{R}), \xi \geq 0$ ou on a noté $H(k)=a(k, 0)$.

Proposition 3 Soient $f, \widehat{f} \in L^{1}(\mathbb{R}), u, \widehat{u} \in L^{\infty}(\mathbb{R})$ solutions entropiques de (PS) correspondant à $f$ et $\widehat{f}$ respectivement, il existe $\alpha \in \operatorname{sign}_{0}^{+}(b(u)-b(\widehat{u}))$ p.p $x \in \mathbb{R}$ telle que :

$$
\int_{\mathbb{R}}(b(u)-b(\widehat{u}))^{+} d x \leq \int_{\mathbb{R}} \alpha(f-\widehat{f})^{+} d x .
$$

Ce résultat est à rapprocher de mes résultats antérieurs [3].

Suivant la théorie générale des équations d'évolution dans les espaces de Banach, on a introduit l'opérateur $A_{b}$ de $L^{1}(\mathbb{R})$ définie par :

$(b(u), v) \in A_{b}, v \in L^{1}(\mathbb{R}) \cap L^{\infty}(\mathbb{R})$ et $u$ est solution entropique de $(P S)$ avec 
$f=v+b(u)$.

On appelle $<<$ bonne-solution $>>$ de $(P E)$ toute fonction $u: Q \rightarrow \mathbb{R}$ mesurable telle que $v=b(u)$ soit solution au sens de la théorie des semi-groupes du problème :

$$
\frac{d v}{d t}+A_{b} v \ni f, \quad v(0)=v_{0} .
$$

Le résultat principal que nous avons développé avec S-Ouaro (cf [7]) est le suivant

Théorème 3 Sous les hypothèses précédentes, étant donné $f \in L^{1}(Q), v_{0} \in L^{1}(\mathbb{R})$, il existe une unique bonne solution de $(P E) v=b(u)$ caractérisée par :

$$
b(u) \in C\left([0, T] ; L^{1}(\mathbb{R})\right), \quad b(u(0))=v_{0} .
$$

et pour tout $\xi \in \mathcal{D}(] 0, T[) \xi \geq 0 b(\widehat{u}) \in \mathcal{D}\left(A_{b}\right)$, il existe $\alpha \in \operatorname{sign}(b(u)-b(\widehat{u}))$ p.p sur $Q$ telle que

$$
\iint_{Q} \alpha\left\{(b(u)-b(\widehat{u})) \xi^{\prime}+\left(f-A_{b} b(\widehat{u})\right) \xi\right\} d x d t \geq 0 .
$$

De plus, les bonnes solutions vérifient le principe de comparaison, plus précisement,

si $f \leq \widehat{f}$ p.p $\operatorname{sur} Q$ et $v \leq \widehat{v}_{0}$ p.p sur $\mathbb{R}$, alors $(b(u) \leq b(\widehat{u})$ p.p sur $Q$ où $u, \widehat{u}$ sont les solutions de $(P E)$ correspondant respectivement aux données $\left(f, v_{0}\right),\left(\widehat{f}, \widehat{v}_{0}\right)$.

Ce résultat est la conséquence directe, d'après le théorème des semi-groupes dans $L^{1}$, du Lemme suivant.

Lemme 1 L'opérateur $A_{b}$ est T-accrétif dans $L^{1}(\mathbb{R})$, à domaine dense dans $L^{1}(\mathbb{R})$ et vérifie la condition d'image, c'est à dire $\forall \lambda>0$, l'image de $I+\lambda A_{b}, R\left(I+\lambda A_{b}\right)$ est dense dans $L^{1}(\mathbb{R})$.

Sous certaines hypothèses restrictives sur les données, la $<<$ bonne-solution $>>$ est solution entropique du problème d'évolution. Nous avons également développer des résultats d'existence et d'unicité de solutions renormalisées du problème considéré.

\section{Bibliographie}

[1] Alt H. W., Luckhauss S., Quasi-linear elliptic-parabolic differential equations, Math. Z., 183, (1983), 311-341.

[2] Bénilan $\mathrm{Ph}$., Touré H., sur l'équation générale $u_{t}=a\left(., u, \varphi(., u)_{x}\right)_{x}$ dans $L^{1}$, I. Etude d'un problème stationnaire, in : Evolution Equation, Proccedings Conference L.S.U., Janvier 1993. Lectures Notes, Vol. 168, (1994), 35-62.

[3] Bénilan Ph., Touré H., sur l'équation générale $u_{t}=a\left(., u, \varphi(., u)_{x}\right)_{x}$ dans $L^{1}$, II. Le problème d'évolution, Ann. Inst. Henri Poincaré, 12, (6), (1995), 727-761.

[4] Bénilan Ph., Wittbold P., On mild and weak solution of elliptic-parabolic problem Adv. in Differential Equations, 1, (6), (1996), 1053-1072.

[5] Carrillo J., Entropy solutions for nonlinear degenerate problems, Arch. Rational Mech. Anal., 147, (1999), 269-361.

[6] Maliki M., Touré H., Solution généralisée locale d'une équation parabolique quasi linéaire dégenérée du second ordre, Ann. Fac-Sa. Toulouse, Vol VII, n 1, (1998), 113-133.

[7] Ouaro S., Touré H., Sur un problème de type elliptique parabolique non linèaire, C. R. Acad. Sci. Pris, Se J334, (2002), 27-30. 